\title{
AN INTEGRATED MODELING METHODOLOGY FOR MATERIAL HANDLING SYSTEMS DESIGN
}

\author{
Gajanana Nadoli \\ Madhav Rangaswami \\ AMHS Design Engineering \\ Intel Corporation \\ 5000 W. Chandler Blvd. \\ Chandler, AZ 85226, U.S.A.
}

\begin{abstract}
We present a modeling methodology for conceptual design and simulation of automated material handling systems (AMHS) in semiconductor manufacturing. This methodology integrates the conceptual design and modeling processes. This results in a reduction in modeling cycle time. The modeling process utilizes a simulator, selected from a suite, which is coupled with a design tool. The design tool, an in-house expert system helps in preliminary analysis of and selection among several alternatives. The design tool then generates appropriate data files to be used by a simulator. A simulator, built using Automod II, represents an AMHS in terms of layout, vehicle control logic, and material flow. We discuss the architecture of a simulator in detail and present a case study. We have developed a set of simulators to handle different vehicle control algorithms.
\end{abstract}

\section{INTRODUCTION}

An Automated Material Handling Systems (AMHS) project consists of four major phases: systems modeling (conceptual design and simulation), detailed design, installation and testing. Modeling of these automated systems is a complex and time-consuming activity. The performance indicators of each building block of the system, though well-understood, do not easily translate into the performance indicators of the entire system. The logic and timing requirements of the control system, and spatial layout nature of the problem result in interactions that are hard to predict without detailed modeling. Reducing the modeling time has a direct impact on the overall project cycle time. For this purpose, we improved our modeling process by developing an integrated environment consisting of a simulator and a design tool.

The AMHS modeling process consists of three iterative steps: requirements analysis, preliminary design, and alternative design analysis. The design tool reduces the time spent for the first two steps while the simulator module reduces the cycle time for the third step. By reducing the designers' time spent in repetitive tasks, this environment enables them to focus on problem solving.

An AMHS project for semiconductor manufacturing systems is typically a part of the design of a new facility (fab). The inputs to the required model are the process flows, operational rules, production volumes, and fab layout. A typical AMHS modeling process requires several iterations. The integrated modeling environment developed in-house, reduces the time required for these iterations.

Section 2 provides an overview of the integrated environment. Section 3 and 4 describe the design tool and simulator respectively. The generic architecture of a simulator is the main focus of this paper, and is covered in Section 5. Section 6 presents a case study of using this environment. Section 7 includes conclusions and scope for future work.

\section{OVERVIEW OF THE INTEGRATED ENVIRONMENT}

Figure 1 shows the structure of the integrated modeling environment. The design tool takes the manufacturing process flow, the fab layout, and the AMHS layout as inputs. The design tool evaluates different AMHS layout arrangements and selects the most practical configuration. The simulator takes the outputs generated from the design tool along with a graphical representation of the system, and builds a simulation model. 
The simulator provides outputs that characterize the system performance.

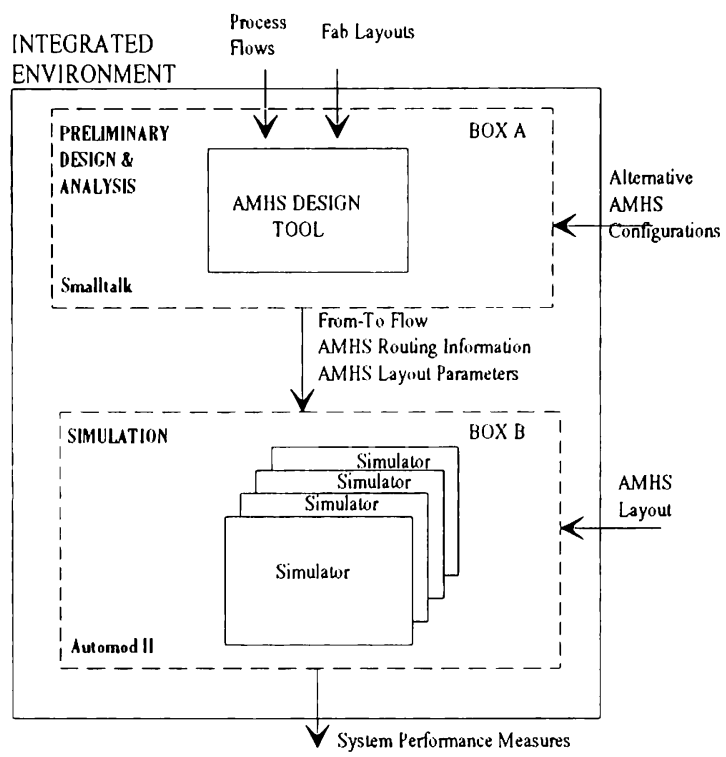

Figure 1: Integrated Modeling Environment

\section{AMHS DESIGN TOOL}

The AMHS design tool (Box A in Figure 1), an in-house expert system, automates the analysis for the conceptual design phase (Nadoli and Macleish, 1993, Kumar et. al., 1993). This phase is characterized by the following: the design task is non-linear, multiple designs are considered at any time, multiple experts take part in the design, design activity takes a long calendar time, and the input information keeps changing. The design tool is activity driven where design activities, represented in the form of a network, force the precedence requirements among the activities. The design activities encoded in the design tool are: process flow inputs, design specifications, fab layout drawing, preliminary calculations, operational assumption specification, storage equipment placement, track drawing, and track loading analysis. Activities such as pricing, billof-material, and scope of work generation are secondary activities used to derive information from a selected design.

The designer prepares the process information file that associates the process steps to production locations (bays) in the fab (in a typical manufacturing scenario, a production lot may require more than 300 distinct processing steps.) The layout of the fab (the location of bays and aisle spaces) is also defined in the form of a file.
Alternatively, the designer may draw the layout using a graphical user interface. Based on the production levels (typically stated in terms of wafer starts/week) the design tool does preliminary calculations on the storage requirements, flow requirements, and activity levels of robotics systems (i.e., storage and transport) in automated storage systems.

The power of the tool comes from its objectoriented architecture and its internal representation of the AMHS track system as a network. The nodes in this network represent the connections to the automated storage and retrieval systems (AS/RS). Using the network (i.e., applying shortest path algorithm and some system specific heuristics), the material flows are assigned to specific paths in the network. This leads to computation of metrics such as lots moved/hr by the system, total distance moved by a lot, total distance moved/hr by the system, and the lots moved per hour through a location. Based on these metrics the tool selects the best AMHS layout among alternative track configurations developed within the design tool. Once a particular layout is chosen based on the preliminary analyses using the design tool, the simulation files generated in the process are passed on to the appropriate simulator.

The need to link the design tool to the simulation necessitated the creation of additional outputs from the design tool:

i) Process Flow: The from-to flows derived from the process flows along with the probability of occurrence of each flow (sampling plans, rework operations, etc.).

ii) Routing: A specialized routing map is a requirement to handle vehicle control in our simulator. The representation provided by the simulation language, did not support this requirement. Hence that map was generated by the design tool.

iii) Layout Characteristics: The track system, depending on the vendor and the control logic, has features such as bridges, loops, bypasses, etc. These features are necessary to define the logic of the operation of the system. The design tool provided an easy method to generate these features. 


\section{AMHS SIMULATOR}

The purpose of the simulator (Box B in Figure 1) is to provide a convenient means of modeling complex AMHS used extensively within Intel's facilities. With this tool, the user can easily model the performance aspects of an AMHS used in a new or existing facility, by only changing certain configuration files and by inputting the layout of the AMHS. The present scope of work includes the modeling of material movement between bays. We have currently developed two different simulators using AutoMod II (AutoSimulations, 1992), which can simulate the effectiveness of two different systems available for different material handling system suppliers. The details of the architecture of the simulator structure will be provided in this paper, along with a case study of its use.

The AMHS simulator logic includes details on Work In Process (WIP) flow mechanisms (storage constraints for certain locations, rules on alternate storage selection), vehicle scheduling features (limits on loaded vehicles moving to a destination, limit on number of empty vehicles called to a location), and vehicular movement and traffic control schemes.

The simulator utilizes inputs provided by the design tool as well as other AMHS information. Such information includes storage configuration details (location on layout, storage capacity, alternate storage locations), and vehicle movement information (including speed, acceleration and deceleration, load pick up and deposit times, etc.) A detailed representation of the AMHS layout (number of loops, locations of bridges, bypasses, spurs, etc.) is also drawn using the graphical capabilities of AutoMod II .

When the information is entered into the model, the user instructs the simulator to start it's runs. The simulator generates reports detailing equipment performance automatically. Typical outputs obtained from the model include:

a. AMHS lot moves per hour,

b. Lot delivery time statistics,

c. Vehicle utilization statistics,

d. Storage capacities at different storage locations,

e. Statistics on time spent in waiting for vehicle pickup,

f. Traffic reports (congestion levels and frequencies) for different locations, etc.
Some reports are generated automatically by the simulation tool while others can be developed by the user. In addition, the simulator allows the user to graphically observe the performance of the AMHS. These graphs can be printed out for future presentations, or can be exported into other presentation packages.

\section{SIMULATOR ARCHITECTURE}

The AMHS simulator consists of 3 main modules:

i) Input module: Here, the simulator reads all the information from configuration files, and initializes state variables. In all, there are four configuration files which define the process flow, track and layout information, and the vehicle routing navigation map.

ii) Control Logic module: In this module of the simulator, details of the controlling logic are defined. Algorithms for WIP control have been developed to limit the quantity of material at a location, and backup storage rules have also been coded within this module. Additionally, algorithms for limiting vehicle movement and traffic control rules have also been included. With these rules, the user can limit the number of vehicles enroute to a destination, or can specify the number of empty vehicles that can be sent to a pickup location. Activities within a bay and within a storage area are also included within this module. Within this module, a complete cycle of lot transactions is simulated, including lot generation, movement from a bay to another bay, and the movement to intermediate storage locations.

iii) Vehicle Scheduler module: Here, the detailed workings of the vehicle controller have been coded. Deadlock avoidance, vehicle claim procedures, and parking lot features have been modeled in detail within this section. Information regarding layout and vehicle navigation obtained from the design tool, are also utilized within this section.

The main power of the simulator is its ease of reuse. With this integrated environment, it is possible for a user to quickly generate a simulation model of a full factory. Before the tool was created, it would take a simulation engineer weeks of effort to build and test out a simulation model. With the introduction of the integrated environment, one can create a detailed simulation 
model in days. The other advantage with the simulator architecture is its ease of change. When new AMHS vendors enter the scene with newer and more sophisticated vehicles, the simulation model can be easily changed. In most cases only the Vehicle Scheduler section of the AMHS Simulator Module would have to be altered. Similarly, we can extend the functionality of the module to include modeling of vehicular movement within a bay.

The simulator is only a template. The actual layout configuration is provided as an input. With this modeling environment, we can model complex material routing schemes which include loops, branches, bypasses, bridges, etc.

\section{CASE STUDY}

This modeling environment has been used to model an AMHS proposed for one of Intel's new fab facilities. The system, depicted in Figure 2, includes more than 20 automated storage locations connected through a track system. Vehicles move along the tracks. The vehicle controller controls the movement of the vehicles and includes logic for traffic control and deadlock avoidance.

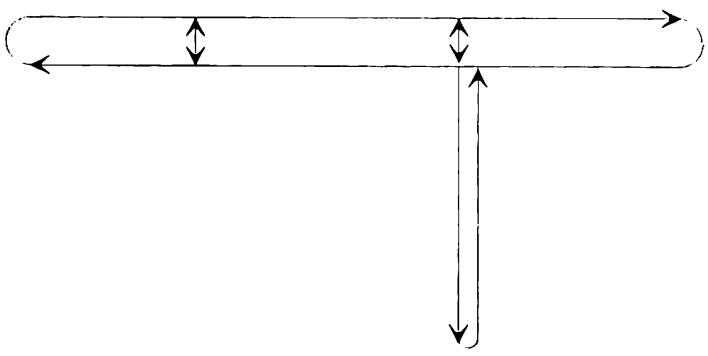

Figure 2: AMHS Layout Schematic

The purpose of the simulation study was to:

a. compare the effect of different vehicle speeds on the performance of the overall AMHS, as measured by the load movement capacity (Figure 3 ) and the load delivery time (Figure 4),

b. determine vehicle utilization as a function of different input loading conditions (Figure 5)

The first step in the modeling process was to input all the necessary information into the design tool. This exercise took less than two days to complete. The next step was to import all the information into the simulator and start the simulation runs. With our typical setup, the complete process takes less than a week of effort. The next three figures show some of the typical outputs obtained from the simulator.

Figure 3 shows the variation of lots moved per hour with input starts per week, for two different vehicle speeds. The graph shows that in order to improve the load carrying capacity of the system, one has to increase the speed of the vehicle from $X \mathrm{fpm}$ to $\mathrm{Y} \mathrm{fpm}$. Without the simulation runs, it would have been very difficult to predict the load carrying capacity of the proposed design.

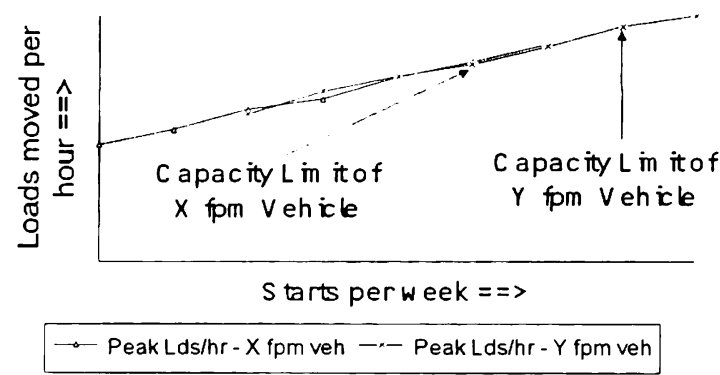

Figure 3: Variation of Lots Moved Per Hour With Input Starts Per Week

Figure 4 depicts the variation in delivery times as a function of starts per week for two different vehicle speeds. The chart shows that there is a threshold starts rate beyond which the delivery times become unacceptably long.

The information on this chart, when coupled with Figure 3 provided us with the key information on system load carrying capacity and delivery times of the proposed design.

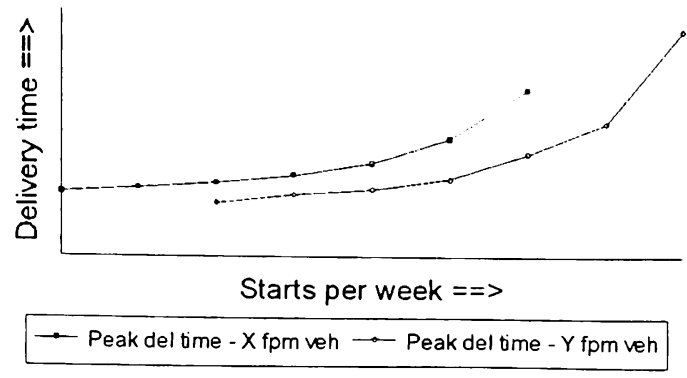

Figure 4: Variation of Delivery Times With Input Starts Per Week 
Figure 5 shows the vehicle utilization as a function of the input start rate. This chart was used to determine the number of vehicles that were required in the system.

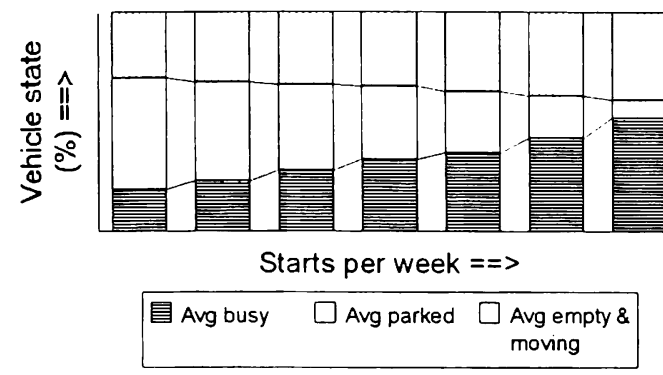

Figure 5: Variation of Vehicle Utilization With Input Starts Per Week

\section{CONCLUSIONS AND FUTURE SCOPE OF WORK}

The integrated simulation environment has proved to be very useful during the design phase of an AMHS. With this tool, we have been able to reduce the modeling cycle time down from weeks to days. The improvement in modeling methodology and the structured model interfaces that we built to support this methodology resulted in this reduction. We estimate that we can develop new simulators in a matter of days for a new vehicle control system. Previously it would take us several weeks to develop such models.

Currently, we have developed two simulators for modeling bay to bay material movement. We plan to increase this library to include simulators for modeling material movement within bays.

Another addition to the simulation module is a statistics extension module. With this, the user would be able to perform powerful statistical analyses, including design of experiments. Such an extension is currently being developed, using AutoStat (AutoSimulations, 1993).

\section{ACKNOWLEDGMENTS}

Many people have assisted us during this effort. We wish to thank Devadas Pillai, Ken St. John, and Kenneth Kryder for providing us with much of the information on the vehicle control systems. We also thank Ken Macleish, Ganapathy Kumar, Don Jacobson, and Court Hilton who were a part of the design tool development effort.

\section{REFERENCES}

AutoSimulations, Inc. 1992. Automod Reference Manual.

AutoSimulations, Inc. 1993. AutoStat Reference Manual.

Nadoli, G., and Macleish, K. 1993. An activityoriented design tool for automated material handling systems design. To appear in the proceedings of the IFIP TC5 Conference, September 12-16, Phoenix, AZ.

Kumar, G., Nadoli, G., Jacobson, D., Hilton, C., and Macleish, K. 1993. AMHS design tool. Proceedings of the Intel Manufacturing for Excellence Conference (Internal Publication).

\section{AUTHOR BIOGRAPHIES}

GAJANANA NADOLI is a Senior Manufacturing Systems Engineer in the Automated Material Handling Systems Design department at Intel Corporation. He received a B.E. in Mechanical Engineering from the University of Mysore (India), and M.A.Sc. and $\mathrm{Ph} . \mathrm{D}$. degrees in Industrial Engineering from the University of Windsor (Canada) and the University of Central Florida, Orlando respectively. His current areas of interests are computer integrated manufacturing, simulation, scheduling systems, object-oriented modeling, and knowledge-based decision support systems.

MADHAV RANGASWAMI is a Senior Industrial Engineer in the Automated Material Handling Systems Design department at Intel Corporation. He received a B.Tech in Mechanical Engineering from Indian Institute of Technology, Delhi (India), an M.E in Mechanical Engineering from the Indian Institute of Science, Bangalore (India) and an M.S in Industrial Engineering from the University of Florida, Gainesville. He has worked in the field of computer simulation for the last eight years, and has been involved with many automation projects in Intel. His current interests are in the fields of simulation, computer integrated manufacturing and factory scheduling systems. 